International Journal of Pure and Applied Mathematics

Volume 84 No. 4 2013, 345-351

ISSN: 1311-8080 (printed version); ISSN: 1314-3395 (on-line version)

url: http://www.ijpam.eu

doi: http://dx.doi.org/10.12732/ijpam.v84i4.4

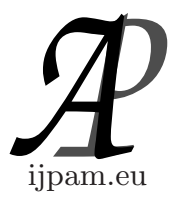

\title{
THE SOLUTION OF EULER-CAUCHY EQUATION EXPRESSED BY DIFFERENTIAL OPERATOR USING LAPLACE TRANSFORM
}

\author{
HwaJoon Kim \\ University of Incheon \\ Incheon, S. KOREA
}

\begin{abstract}
It is well known fact that the Laplace transform is useful in solving linear ordinary differential equations with constant coefficients such as free/forced oscillations, but in the case of differential equation with variable coefficients is not. In here, we would like to propose the Laplace transform of Euler-Cauchy equation with variable coefficients, and find the solution of Euler-Cauchy equation represented by the differential operator using Laplace transform. The purpose of this research is to make an application to its difference equation and oscillation.
\end{abstract}

AMS Subject Classification: 44A10, 34A12

Key Words: Euler-Cauchy equation, variable coefficients, Laplace transform, z transform, oscillations

\section{Introduction}

Euler-Cauchy equations are ODEs of the form $t^{2} y^{\prime \prime}+a t y^{\prime}+b y=0$ with given constants $a$ and $b$ and unknown $y(t)$ (see [8]). The equation appears in a numbers of physics and engineering applications, such as when solving Laplace's equation in a polar coordinates, describing time-harmonic vibrations of a thin

Received: October 29, 2012

(c) 2013 Academic Publications, Ltd. url: www.acadpubl.eu 
elastics rod, boundary value problem in spherical coordinates and so on.

Several researches have been pursued for the stability, the oscillation (see [1]-[4], [11]) and Laplace transform (see [5], [9]-[10]) of differential equations with variable coefficients. Through many trials, they found that the Laplace method is not appropriate for ODEs with variable coefficients, but many applications to its difference equation, the discrete equivalent of differential equations, are still stayed. Using the property of $L\{t f(t)\}=-F^{\prime}(s)$ for $L(f)=F(S)$ and $L(f)$ is Laplace transform of a function $f$, we would like to propose the Laplace transform of Euler-Cauchy equation, and find the solution of Euler-Cauchy equation represented by differential operator using Laplace transform. The purpose of this research is to make an application to its difference equation/z transform and oscillation.

In this paper, we have showed that a solution of Euler-Cauchy equation can be expressed by

$$
\begin{gathered}
y=m y(0) e^{\frac{a}{2} t} \cos \sqrt{\frac{b}{m}-\frac{a^{2}}{4}} t \\
+\left\{a y(0)(m / 2-1)+m y^{\prime}(0)\right\}\left(1 / \sqrt{\frac{b}{m}-\frac{a^{2}}{4}}\right) e^{\frac{a}{2} t} \sin \sqrt{\frac{b}{m}-\frac{a^{2}}{4}} t
\end{gathered}
$$

where $d / d s=m$.

\section{The Solution of Euler-Cauchy Equation using Laplace Transform}

Euler-Cauchy equations have the form $t^{2} y^{\prime \prime}+a t y^{\prime}+b y=0$, and let us check the Laplace transform of Euler-Cauchy equation represented by the second derivative. Remark that

and

$$
L\left(t y^{\prime}\right)=-Y-s \frac{d Y}{d s}
$$

$$
\begin{aligned}
L\left(t^{2} y^{\prime \prime}\right) & =\frac{d^{2}}{d s^{2}}\left[s^{2} Y-s y(0)-y^{\prime}(0)\right] \\
& =-\frac{d}{d s}\left[2 s Y+s^{2} \frac{d Y}{d s}-y(0)\right] \\
& =2 Y+4 s \frac{d Y}{d s}+s^{2} \frac{d^{2} Y}{d s^{2}} .
\end{aligned}
$$

Hence, the Laplace transform of Euler-Cauchy equation is expressed by

$$
s^{2} \frac{d^{2} Y}{d s^{2}}+(4 s-a s) \frac{d Y}{d s}+(b-a+2) Y=0
$$


where $Y=L(y)=F(s)$ and $L(y)$ is the Laplace transform of $y$.

By the Frobenius Method (see [7], p. 182), the Euler-Cauchy equation has a basis of the form $x^{r_{1}} a_{0}$ and $x^{r_{2}} b_{0}$ where $a_{0}$ and $b_{0}$ are constants, and $r^{2}+(3-a) r+(b-a+2)=0$.

Next we would like to apply the method of reduction of order credited J. L. Lagrange to the equation $(*)$.

Lemma 1. The equation $\left({ }^{*}\right)$ has the form of a basis $y_{1}$ and

$$
y_{1} \int s^{a-4} / y_{1}^{2} d s
$$

of the solutions. In particular, if $b=(a-1)^{2} / 4$, then $\left(^{*}\right)$ has bases

$$
s^{\frac{a-3}{2}}, s^{a-3 / 2} \ln |s| \text {. }
$$

Proof. We assume a solution $y_{1}$ of the equation on an open interval $I$ to be known and want to find a basis. Let us find a second solution $y_{2}$. We substitute

$$
y=y_{2}=u y_{1}, y^{\prime}=u^{\prime} y_{1}+u y_{1}^{\prime}, y^{\prime \prime}=u^{\prime \prime} y_{1}+2 u^{\prime} y_{1}^{\prime}+u y_{1}^{\prime \prime}
$$

into the equation $(*)$. This gives

$$
u^{\prime \prime} y_{1} s^{2}+u^{\prime}\left\{2 y_{1}^{\prime} s^{2}+(4-a) s y_{1}\right\}+u\left\{y_{1}^{\prime \prime} s^{2}+(4-a) s y_{1}^{\prime}+(b-a+2) y_{1}\right\}=0 .
$$

As we divide by $y_{1} s^{2}$ and put $u^{\prime}=v$ for $y_{1}^{\prime \prime} s^{2}+(4-a) s y_{1}^{\prime}+(b-a+2) y_{1}=0$, we have

$$
v^{\prime}+v\left\{\frac{2 y_{1}^{\prime}}{y_{1}}+\frac{4-a}{s}\right\}=0 .
$$

By the the simple calculation, we get

$$
u=\int s^{a-4} / y_{1}^{2} d s
$$

and so

$$
y_{2}=y_{1} u=y_{1} \int s^{a-4} / y_{1}^{2} d s .
$$

Since $u>0$, the quotient $y_{2} / y_{1}=u=\int v d s$ cannot be constant, so that $y_{1}$ and $y_{2}$ form a basis of solutions.

Next, the second statement is immediately followed from

$$
y_{1}=s^{\frac{a-3}{2}} .
$$


Recall that

$$
L^{-1}\left\{\frac{1}{(s-a)^{2}+w^{2}}\right\}=\frac{1}{w} e^{a t} \text { sinwt }
$$

and

$$
L^{-1}\left\{\frac{s-a}{(s-a)^{2}+w^{2}}\right\}=e^{a t} \cos w t
$$

for $L^{-1}$ is the inverse Laplace transform. Now that let us go to the theorem 2 .

Theorem 2. The solution of Euler-Cauchy equation $t^{2} y^{\prime \prime}+a t y^{\prime}+b y=0$ can be expressed by

$$
\begin{gathered}
y=m y(0) e^{\frac{a}{2} t} \cos \sqrt{\frac{b}{m}-\frac{a^{2}}{4}} t \\
+\left\{a y(0)(m / 2-1)+m y^{\prime}(0)\right\}\left(1 / \sqrt{\frac{b}{m}-\frac{a^{2}}{4}}\right) e^{\frac{a}{2} t} \sin \sqrt{\frac{b}{m}-\frac{a^{2}}{4}} t
\end{gathered}
$$

where $d / d s=m$.

Proof. Taking Laplace transform on both sides of the above equation, we get

$$
L\left\{t^{2} \frac{d y^{2}}{d t^{2}}\right\}+a L\left\{t \frac{d y}{d t}\right\}+b L(y)=0 .
$$

Using the derivative property of Laplace transform, we have

$$
\frac{d^{2}}{d s^{2}} L\left(\frac{d y^{2}}{d t^{2}}\right)-a \frac{d}{d s} L\left(\frac{d y}{d t}\right)+b L(y)=0 .
$$

By the Laplace transform of derivatives, we obtain

$$
\frac{d^{2}}{d s^{2}}\left[s^{2} Y-s y(0)-y^{\prime}(0)\right]-a \frac{d}{d s}[s Y-y(0)]+b Y=0
$$

for $Y=L(y)=F(s)$. Let us put $d / d s=m$. Then

$$
m^{2}\left[s^{2} Y-s y(0)-y^{\prime}(0)\right]-a m[s Y-y(0)]+b Y=0
$$

and arranging the equation,

$$
Y=\frac{s m^{2} y(0)+m^{2} y^{\prime}(0)-a m y(0)}{m s^{2}-a m s+b}
$$

for $m s^{2}-a m s+b \neq 0$. What we want to find is $y(t)$ for all values of $t$. We have

$$
y=L^{-1}(Y)
$$




$$
\begin{aligned}
= & L^{-1}\left[\frac{s m^{2} y(0)+m^{2} y^{\prime}(0)-a m y(0)}{m s^{2}-a m s+b}\right] \\
= & L^{-1}\left[\frac{s m^{2} y(0)+m^{2} y^{\prime}(0)}{m s^{2}-a m s+b}\right]+L^{-1}\left[\frac{-a m y(0)}{m s^{2}-a m s+b}\right] \\
= & L^{-1}\left[\frac{s m y(0)+m y^{\prime}(0)}{s^{2}-a s+b / m}\right]-a L^{-1}\left[\frac{y(0)}{s^{2}-a s+b / m}\right] \\
= & L^{-1}\left[\frac{\left(s-\frac{a}{2}\right) m y(0)+\frac{a m}{2} y(0)+m y^{\prime}(0)}{\left(s-\frac{a}{2}\right)^{2}+\frac{b}{m}-\frac{a^{2}}{4}}\right] \\
& -a y(0) L^{-1}\left[\frac{1}{\left(s-\frac{a}{2}\right)^{2}+\frac{b}{m}-\frac{a^{2}}{4}}\right] .
\end{aligned}
$$

As we scan a table of Laplace transforms, we see that

$$
\begin{aligned}
y= & m y(0) e^{\frac{a}{2} t} \cos \sqrt{\frac{b}{m}-\frac{a^{2}}{4}} t \\
& +m\left\{\frac{a}{2} y(0)+y^{\prime}(0)\right\}\left(1 / \sqrt{\frac{b}{m}-\frac{a^{2}}{4}}\right) e^{\frac{a}{2} t} \sin \sqrt{\frac{b}{m}-\frac{a^{2}}{4}} t \\
& -a y(0)\left(1 / \sqrt{\frac{b}{m}-\frac{a^{2}}{4}}\right) e^{\frac{a}{2} t} \sin \sqrt{\frac{b}{m}-\frac{a^{2}}{4}} t,
\end{aligned}
$$

and hence, we have

$$
\begin{aligned}
y= & m y(0) e^{\frac{a}{2} t} \cos \sqrt{\frac{b}{m}-\frac{a^{2}}{4}} t \\
& +\left\{a y(0)(m / 2-1)+m y^{\prime}(0)\right\}\left(1 / \sqrt{\frac{b}{m}-\frac{a^{2}}{4}}\right) e^{\frac{a}{2} t} \sin \sqrt{\frac{b}{m}-\frac{a^{2}}{4}} t .
\end{aligned}
$$

If the continuous signal $f(t)$ is sampled at intervals of time, $T$, we obtain a sequence of sampled values $f(k), k \in N$. Since the alternated representation of the equation $(*)$ is

$$
Y=\frac{s m^{2} y(0)+m^{2} y^{\prime}(0)-a m y(0)}{m s^{2}-a m s+b},(d / d s=m)
$$

it is clear that the equation

$$
(* *) \approx T f^{*}(t)=T \sum_{k=0}^{\infty} f(k) \delta(t-k T)
$$

where $f^{*}(t)$ is a weighted impulses. On the other hand, the result of (see [6]) shows that if the Euler-Cauchy equation has a complex characteristic roots, it is oscillatory on $T$. 


\section{References}

[1] R.B. Chukleva, A.B. Dishliev, K.G. Dishlieva, Stability of the differential equations with variable structue and non fixed impulsive moments using sequences of Lyapunov's functions, International Journal of Differential Equations and Applications, 11, No. 1 (2012), 57-80.

[2] K.G. Dishlieva, A.B. Dishliev, S.A. Petkova, Death of the solutions of systems differential equations with variable structure and impulses, International Journal of Differential Equations and Applications, 11, No. 3 (2012), 169-181.

[3] M.B. Dimitrova, V.I. Donev, Oscillation criteria for the solutions of a first order neutral nonconstant delay impulsive differential equations with variable coefficients, International Journal of Pure and Applied Mathematics, 73, No. 1 (2011), 13-28.

[4] M.B. Dimitrova, V.I. Donev, On the nonoscillation and oscillation of the solutions of a first order neutral nonconstant delay impulsive differential equations with variable or oscillating coefficients, International Journal of Pure and Applied Mathematics, 73, No. 1 (2011), 111-128.

[5] Tarig M. Elzaki, Salih M. Ezaki, On the Elzaki transform and ordinary differential equation with variable coefficients, The Advances in Theoretical and Applied Mathematics, 6, No. 1 (2011), 41-46.

[6] S. Huff, G. Olumolode, N. Pennington, A. Peterson, Oscillation of an EulerCauchy daynamic equation, In: Proceedings on the Fourth International Conference on Dynamical Systems and Differential Equations, May 24-27 (2002), 423-431.

[7] E. Kreyszig, Advanced Engineering Mathematics, John Willy \& Sons, Inc., New York (2012).

[8] R. Kent Nagle, Edward B. Saff, A.D. Snider, Fundamental of Differential Equation, Pearson, Inc., Boston (1989).

[9] A. Kumar, D.K. Jaiswal, N. Kumar, Analytical solutions to onedimensional advection-diffusion equation with variable coefficients in semiinfinite media, Journal of Hydrology, 380, No-s: 3-4 (2009), 330-337.

[10] S.R.S. Varadhan, On the behavior of the fundamental solution of the heat equation with variable coefficients, Comunication on Pure and Applied Mathematics (1967), 431-55. 
[11] Y. Zhang, New grammian solutions for a variable-coefficient MKPI equation, International Journal of Pure and Applied Mathematics, 79, No. 3 (2012), 375-379. 
